\title{
Approximation Properties for Stancu Type $q$-Baskakov- Kantorovich Operators
}

\author{
Çiğdem Atakut and İbrahim Büyükyazıc** \\ Faculty of Science, Department of Mathematics, Ankara University, Tandogan 06100, Ankara, Turkey
}

Received: 14 Jun. 2013, Revised: 10 Oct. 2013, Accepted: 11 Oct. 2013

Published online: 1 Jan. 2014

\begin{abstract}
In this paper, we give an interesting generalization of the Stancu type Baskakov-Kantorovich operators based on the qintegers and investigate their approximation properties. Also, we obtain the estimates for the rate of convergence for a sequence of them by the weighted modulus of smoothness.
\end{abstract}

Keywords: $q-$ integer, $q-$ Baskakov-Kantorovich operators, Baskakov-Kantorovich-Stancu operators, weighted spaces, rate of convergence, weighted modulus of smoothness.

\section{Introduction}

In recent years, due to the intensive development of $q-$ calculus, generalizations of some operators related to $q-$ calculus have emerged (see $[2,3,7,12-16])$. Aral and Gupta defined $q-$ generalization of the Baskakov operator and investigated approximation properties of these operators in [3]. In [13], Gupta and Radu introduced the Baskakov- Kantorovich operators based on $q$-integers and investigated their weighted statistical approximation properties. They also proved some direct estimations for error using weighted modulus of smoothness in case $0<q<1$. In recent study Büyükyazıcı and Atakut [7] introduced a new Stancu type generalization of $q-$ Baskakov operator is defined as

$L_{n}^{\alpha, \beta}(f ; q, x)=\sum_{k=0}^{\infty} q^{\frac{k(k-1)}{2}} \frac{D_{q}^{k}\left(\varphi_{n}(x)\right)}{[k]_{q} !}(-x)^{k} f\left(\frac{1}{q^{k-1}} \frac{[k]_{q}+q^{k-1} \alpha}{[n]_{q}+\beta}\right)$

where $0 \leq \alpha \leq \beta, q \in(0,1), f \in C[0, \infty)$ and the following conditions are provided:

Let $\left\{\varphi_{n}\right\}(n=1,2, \ldots) \varphi_{n}: \mathbb{R} \rightarrow \mathbb{R}$ be a sequence which is satisfying following conditions,

(i) $\varphi_{n}(n=1,2, \ldots), k-$ times continuously $q-$ differentiable any closed interval $[0, A]$,

(ii) $\varphi_{n}(0)=1,(n=1,2, \ldots)$,

(iii) for all $x \in[0, A]$, and $(k=0,1, \ldots ; n=1,2, \ldots)$, $(-1)^{k} D_{q}^{k}\left(\varphi_{n}(x)\right) \geq 0$, (iv) there exists a positive integer $m(n)$, such that

$$
D_{q}^{k}\left(\varphi_{n}(x)\right)=-[n]_{q} D_{q}^{k-1} \varphi_{m(n)}(x),(k=1,2, \ldots ; n=1,2, \ldots),
$$

(v) $\lim _{n \rightarrow \infty} \frac{[n]_{q}}{[m(n)]_{q}}=1$.

Now, to explain the construction of the new $q-$ operators, we mention some basic definitions of $q-$ calculus and Lemma.

Let $q>0$. For each nonnegative integer $n$, we define the $q$ - integer $[n]_{q}$ as

$$
[n]_{q}=\left\{\begin{array}{lll}
\left(1-q^{n}\right) /(1-q) & \text { if } & q \neq 1 \\
n & \text { if } & q=1
\end{array}\right.
$$

and the $q-$ factorial $[n]_{q}$ ! as

$$
[n]_{q} !=\left\{\begin{array}{lll}
{[n]_{q}[n-1]_{q} \cdots[1]_{q}} & \text { if } & n \geq 1 \\
1 & \text { if } & n=0
\end{array}\right.
$$

For the integers $n$ and $k$, with $0 \leq k \leq n$, the $q$ - binomial coefficients are then defined as follows (see [15]):

$$
\left[\begin{array}{l}
n \\
k
\end{array}\right]_{q}=\frac{[n]_{q} !}{[k]_{q} ![n-k]_{q} !} .
$$

Note that the following relation is satisfied

$$
[n]_{q}=[n-1]_{q}+q^{n-1} .
$$

\footnotetext{
*Corresponding author e-mail: ibuyukyazici@gmail.com
} 
Definition 1.1. The $q$ - derivative of a function $f$ with respect to $x$ is

$D_{q}(f(x))=\frac{f(q x)-f(x)}{q x-x}, x \neq 0 ; D_{q}(f(0))=\lim _{x \rightarrow 0} D_{q}(f(x))$

which is also known as the Jackson derivative. High $q-$ derivatives are

$D_{q}^{0}(f(x))=f(x), D_{q}^{n}(f(x))=D_{q}\left(D_{q}^{n-1}(f(x))\right), n=1,2,3, \ldots$

Note that as $q \rightarrow 1$, the $q$ - derivative approach the usual derivative.

Definition 1.2. The $q$-integration is defined as

$$
\int_{0}^{a} f(t) d_{q} t=(1-q) a \sum_{j=0}^{\infty} f\left(q^{j} a\right) q^{j} \quad, a>0 .
$$

Over a general interval $[a, b], 0<a<b$, one defines

$$
\int_{a}^{b} f(t) d_{q} t=\int_{0}^{b} f(t) d_{q} t-\int_{0}^{a} f(t) d_{q} t
$$

Definition 1.3. Let $f(x)$ be a continuous function on some interval $[a, b]$ and $c \in(a, b)$. Jackson's $q$ - Taylor formula (see $[14,15])$ is given by

$$
f(x)=\sum_{k=0}^{\infty} \frac{\left(D_{q}^{k} f\right)(c)}{[k]_{q} !}(x-c)_{q}^{k}
$$

where $(x-c)_{q}^{k}=\prod_{i=0}^{k-1}\left(x-c q^{i}\right)$.

First we need the following auxiliary result. Throughout the paper, we use $e_{i}$ the test functions defined by $e_{i}(t):=t^{i}$ for every integer $i \geq 0$.

Lemma 1.4. ( [7]) Let $L_{n}^{\alpha, \beta}$ be defined by (1). Then the following identities hold:

$$
L_{n}^{\alpha, \beta}\left(e_{0} ; q, x\right)=1,
$$

$$
L_{n}^{\alpha, \beta}\left(e_{1} ; q, x\right)=\frac{[n]_{q}}{[n]_{q}+\beta} x+\frac{\alpha}{[n]_{q}+\beta}
$$

$L_{n}^{\alpha, \beta}\left(e_{2} ; q, x\right)=\frac{[n]_{q}[m(n)]_{q}}{q\left([n]_{q}+\beta\right)^{2}} x^{2}+\frac{[n]_{q}(2 \alpha+1)}{\left([n]_{q}+\beta\right)^{2}} x+\frac{\alpha^{2}}{\left([n]_{q}+\beta\right)^{2}}$.

\section{Some properties of Stancu type $q$-Baskakov-Kantorovich operators}

Let $\left\{\varphi_{n}\right\}$ be a sequence of real functions on $\mathbb{R}_{+}=[0, \infty)$ which are $k$ - times continuously $q$ - differentiable on $\mathbb{R}_{+}$ satisfying following conditions: (c1) $\varphi_{n}(0)=1,(n=1,2, \ldots)$,

(c2) for $k \in \mathbb{N}_{0}=\mathbb{N} \cup\{0\}$ and $n \in \mathbb{N}$, $(-1)^{k} D_{q}^{k}\left(\varphi_{n}(x)\right) \geq 0, x \in \mathbb{R}_{+}$,

(c3) there exists a positive integer $m(n)$, such that

$$
D_{q}^{k}\left(\varphi_{n}(x)\right)=-[n]_{q} D_{q}^{k-1} \varphi_{m(n)}(x) ; \quad k, n \in \mathbb{N}
$$

(c4) $\lim _{n \rightarrow \infty} \frac{[n]_{q}}{[m(n)]_{q}}=1$.

In this paper, under the conditions $(c 1)-(c 4)$, we definition a new generalization of Stancu type $q$-Baskakov-Kantorovich operators as following

$$
\begin{gathered}
L_{n}^{*(\alpha, \beta)}(f ; q, x)=\left([n]_{q}+\beta\right) \sum_{k=0}^{\infty} q^{\frac{k(k-1)}{2}} \frac{D_{q}^{k}\left(\varphi_{n}(x)\right)}{[k]_{q} !}(-x)^{k} \\
\\
\int_{q\left(\frac{[k]_{q}+q^{k-1} \alpha}{[n]_{q}+\beta}\right)}^{\frac{[k+1]_{q}+q^{k} \alpha}{[n]_{q}+\beta}} f\left(q^{-k+1} t\right) d_{q} t
\end{gathered}
$$

where $x \in \mathbb{R}_{+}, n \in \mathbb{N}, 0 \leq \alpha \leq \beta$.

Remark 2.1. Let $x \in \mathbb{R}_{+}$. If $\varphi_{n}(x)=e_{q}^{-[n]_{q} x}$, then for all $k, n \in \mathbb{N}$ we have $D_{q}^{k}\left(\varphi_{n}(x)\right)=(-1)^{k}[n]_{q}^{k} e_{q}^{-[n]_{q} x}$. In this case the operators $L_{n}^{*(\alpha, \beta)}$ reduce to Stancu type $q$-Szász-Kantorovich operators given as follows:

$$
\begin{gathered}
L_{n}^{*(\alpha, \beta)}(f, q, x)=\left([n]_{q}+\beta\right) e_{q}^{-[n]_{q} x} \sum_{k=0}^{\infty} q^{\frac{k(k-1)}{2}} \frac{\left([n]_{q} x\right)^{k}}{[k]_{q} !} \\
\int_{q\left(\frac{[k] q+q^{k-1} \alpha}{[n]^{+\beta}}\right)}^{\frac{[k+1]_{q}+q^{k} \alpha}{[n]_{q}+\beta}} f\left(q^{-k+1} t\right) d_{q} t,
\end{gathered}
$$

where $e_{q}^{-[n]_{q} x}$ is $q$-analogues of the exponential function defined by

$$
e_{q}^{x}=\sum_{k=0}^{\infty} q^{\frac{k(k-1)}{2}} \frac{x^{k}}{[k]_{q} !} .
$$

In each of the following theorems, we assume that $q=$ $q_{n}$, where $\left\{q_{n}\right\}$ is a sequence of real numbers such that $0<q_{n}<1$ for all $n$ and $\lim _{n \rightarrow \infty} q_{n}=1$.

Now we give the following Lemmas, which are necessary to prove our theorems:

Lemma 2.2. The following relations are satisfied:

$$
\int_{q\left(\frac{[k]_{q}+q^{k-1} \alpha}{[n]_{q}+\beta}\right)}^{\frac{[k+1]_{q}+q^{k} \alpha}{[n]_{q}+\beta}} d_{q} t=\frac{1}{[n]_{q}+\beta},
$$




$$
\int_{q\left(\frac{[k]_{q}+q^{k-1} \alpha}{[n]_{q}+\beta}\right)}^{\frac{[k+1]_{q}+q^{k} \alpha}{[n]_{q}+\beta}} t d_{q} t=\frac{[2]_{q}[k]_{q}+q^{k}(1+2 \alpha)}{[2]_{q}\left([n]_{q}+\beta\right)^{2}},
$$

$$
\int_{\left(\frac{[k]_{q}+q^{k-1} \alpha}{[n]_{q}+\beta}\right)}^{\frac{[k+1]_{q}+q^{k} \alpha}{[n]_{q}+\beta}} t^{2} d_{q} t=\frac{[3]_{q}[k]_{q}^{2}+q^{k}[k]_{q}\left((1+3 \alpha)[2]_{q}+1\right)+\left(1+3 \alpha+3 \alpha^{2}\right) q^{2 k}}{[3]_{q}\left([n]_{q}+\beta\right)^{3}}
$$

Proof. From properties of $q$-analogue integration, by simple computation we obtain (7-9).

By the following Lemma Korovkin's conditions are satisfied.

Lemma 2.3. For all $x \in \mathbb{R}_{+}, n \in \mathbb{N}, \quad \alpha, \beta \geq 0$ and $0<q<1$, we have

$$
\begin{aligned}
L_{n}^{*(\alpha, \beta)}\left(e_{0} ; q, x\right)=1 \\
L_{n}^{*(\alpha, \beta)}\left(e_{1} ; q, x\right)=\frac{[n]_{q}}{[n]_{q}+\beta} x+\frac{q(1+2 \alpha)}{[2]_{q}\left([n]_{q}+\beta\right)}, \\
L_{n}^{*(\alpha, \beta)}\left(e_{2} ; q, x\right)=\frac{[n]_{q}[m(n)]_{q}}{q\left([n]_{q}+\beta\right)^{2}} x^{2} \\
+\frac{[n]_{q}\left[[3]_{q}+q\left((1+3 \alpha)[2]_{q}+1\right)\right]}{x} x \\
+\frac{q^{2}(1+3 \alpha]_{q}\left([n]_{q}+\beta\right)^{2}}{[3]_{q}\left([n]_{q}+\beta\right)^{2}} .
\end{aligned}
$$

Proof. From definition (6) and the identities(3) and (7), we can easily obtain

$$
\begin{aligned}
L_{n}^{*(\alpha, \beta)}\left(e_{0} ; q, x\right)= & \left([n]_{q}+\beta\right) \sum_{k=0}^{\infty} q^{\frac{k(k-1)}{2}} \frac{D_{q}^{k}\left(\varphi_{n}(x)\right)}{[k]_{q} !}(-x)^{k} \\
& \frac{[k+1]_{q}+q^{k} \alpha}{[n]_{q}+\beta} \\
& q\left(\frac{[k]_{q}+q^{k-1} \alpha}{[n]_{q}+\beta}\right) \\
= & L_{n}^{\alpha, \beta}\left(e_{0} ; q, x\right)=1 .
\end{aligned}
$$

Now for $e_{1}$,from (3), (4) and (8) we can write

$$
\begin{aligned}
= & \sum_{k=0}^{\infty} q^{\frac{k(k-1)}{2}} \frac{D_{q}^{k}\left(\varphi_{n}(x)\right)}{[k]_{q} !} \sum_{k=0}^{\infty} q^{\frac{k(k-1)}{2}} \frac{[k]_{q}+q^{k-1} \alpha}{q^{k-1}\left([n]_{q}+\beta\right)} \\
& -\sum_{k=0}^{\infty} q^{\frac{k(k-1)}{2}} \frac{D_{q}^{k}\left(\varphi_{n}(x)\right)}{[k]_{q} !} \frac{q^{k-1} \alpha}{q^{k-1}\left([n]_{q}+\beta\right)} \\
& +\frac{q(1+2 \alpha)}{[2]_{q}\left([n]_{q}+\beta\right)} \sum_{k=0}^{\infty} q^{\frac{k(k-1)}{2}} \frac{D_{q}^{k}\left(\varphi_{n}(x)\right)}{[k]_{q} !}(-x)^{k} \\
= & L_{n}^{\alpha, \beta}\left(e_{1} ; q, x\right)-\frac{\alpha}{[n]_{q}+\beta} L_{n}^{\alpha, \beta}\left(e_{0} ; q, x\right)+\frac{q(1+2 \alpha)}{[2]_{q}\left([n]_{q}+\beta\right)} L_{n}^{\alpha, \beta}\left(e_{0} ; q, x\right) \\
= & \frac{[n]_{q}}{[n]_{q}+\beta} x+\frac{q(1+2 \alpha)}{[2]_{q}\left([n]_{q}+\beta\right)} .
\end{aligned}
$$

The finally, for $e_{2}$, we use (3), (4), (5) and (9), one has

$L_{n}^{*(\alpha, \beta)}\left(e_{2} ; q, x\right)=\left([n]_{q}+\beta\right) \sum_{k=0}^{\infty} q^{\frac{k(k-1)}{2}} \frac{D_{q}^{k}\left(\varphi_{n}(x)\right)}{[k]_{q} !}(-x)^{k}$

$$
\begin{aligned}
& \frac{[k+1]_{q}+q^{k} \alpha}{[n]_{q}+\beta} \\
& q^{-2 k+2} t^{2} d_{q} t \\
& q\left(\frac{[k]_{q}+q^{k-1} \alpha}{[n]_{q}+\beta}\right) \\
& =\sum_{k=0}^{\infty} q^{\frac{k(k-1)}{2}} \frac{D_{q}^{k}\left(\varphi_{n}(x)\right)}{[k]_{q} !}(-x)^{k}\left(\frac{[k]_{q}+q^{k-1} \alpha}{q^{k-1}\left([n]_{q}+\beta\right)}\right)^{2} \\
& -2 \sum_{k=0}^{\infty} q^{\frac{k(k-1)}{2}} \frac{D_{q}^{k}\left(\varphi_{n}(x)\right)}{[k]_{q} !}(-x)^{k} q^{-2 k+2} q^{k-1} \alpha \frac{[k]_{q}+q^{k-1} \alpha}{\left([n]_{q}+\beta\right)^{2}} \\
& +\sum_{k=0}^{\infty} q^{\frac{k(k-1)}{2}} \frac{D_{q}^{k}\left(\varphi_{n}(x)\right)}{[k]_{q} !}(-x)^{k} q^{-2 k+2} \frac{q^{2 k-2} \alpha^{2}}{\left([n]_{q}+\beta\right)^{2}} \\
& +\sum_{k=0}^{\infty} q^{\frac{k(k-1)}{2}} \frac{D_{q}^{k}\left(\varphi_{n}(x)\right)}{[k]_{q} !}(-x)^{k} q^{-2 k+2}\left((1+3 \alpha)[2]_{q}+1\right) \frac{[k]_{q}+q^{k-1} \alpha}{[3]_{q}\left([n]_{q}+\beta\right)^{2}} \\
& -\sum_{k=0}^{\infty} q^{\frac{k(k-1)}{2}} \frac{D_{q}^{k}\left(\varphi_{n}(x)\right)}{[k]_{q} !}(-x)^{k} q^{-2 k+2} q^{k} q^{k-1} \alpha \frac{\left((1+3 \alpha)[2]_{q}+1\right)}{[3]_{q}\left([n]_{q}+\beta\right)^{2}} \\
& +\sum_{k=0}^{\infty} q^{\frac{k(k-1)}{2}} \frac{D_{q}^{k}\left(\varphi_{n}(x)\right)}{[k]_{q} !}(-x)^{k} \frac{q^{2}\left(1+3 \alpha+3 \alpha^{2}\right)}{[3]_{q}\left([n]_{q}+\beta\right)^{2}} \\
& =L_{n}^{\alpha, \beta}\left(e_{2} ; q, x\right)-\frac{2 \alpha}{[n]_{q}+\beta} L_{n}^{\alpha, \beta}\left(e_{1} ; q, x\right)+\frac{\alpha^{2}}{\left([n]_{q}+\beta\right)^{2}} L_{n}^{\alpha, \beta}\left(e_{0} ; q, x\right) \\
& +\frac{q\left((1+3 \alpha)[2]_{q}+1\right)}{[3]_{q}\left([n]_{q}+\beta\right)} L_{n}^{\alpha, \beta}\left(e_{1} ; q, x\right)-q \alpha \frac{\left((1+3 \alpha)[2]_{q}+1\right)}{[3]_{q}\left([n]_{q}+\beta\right)^{2}} L_{n}^{\alpha, \beta}\left(e_{0} ; q, x\right) \\
& +\frac{q^{2}\left(1+3 \alpha+3 \alpha^{2}\right)}{[3]_{q}\left([n]_{q}+\beta\right)^{2}} L_{n}^{(\alpha, \beta)}\left(e_{0} ; q, x\right) \\
& =\frac{[n]_{q}[m(n)]_{q}}{q\left([n]_{q}+\beta\right)^{2}} x^{2} \\
& +\frac{[n]_{q}\left([3]_{q}+q\left((1+3 \alpha)[2]_{q}+1\right)\right)}{[3]_{q}\left([n]_{q}+\beta\right)^{2}} x+\frac{q^{2}\left(1+3 \alpha+3 \alpha^{2}\right)}{[3]_{q}\left([n]_{q}+\beta\right)^{2}} .
\end{aligned}
$$


This completes the proof of Lemma 2.3.

Using above Lemma, we can obtain following theorem.

Theorem 2.4. Let $q_{n} \in(0,1)$ and $\lim _{n \rightarrow \infty} q_{n}=1$. Then the sequence $\left\{L_{n}^{*(\alpha, \beta)}\left(f ; q_{n},.\right)\right\}$ converges to $f$ uniformly on $[0, A]$ for each $f \in C\left(\mathbb{R}_{+}\right)$and $A>0$.

\section{Rate of convergence}

$B_{\rho_{\gamma}}\left(\mathbb{R}_{+}\right)$, the weighted space of real valued functions $f$ defined on $\mathbb{R}_{+}$with the property $|f(x)| \leq M_{f} \rho_{\gamma}(x)$ where $\rho_{\gamma}(x)=1+x^{\gamma+2}$ and $M_{f}$ is constant depending on the function $f$. We also consider the weighted subspace $C_{\rho_{\gamma}}\left(\mathbb{R}_{+}\right)$of $B_{\rho_{\gamma}}\left(\mathbb{R}_{+}\right)$given by

$$
C_{\rho_{\gamma}}\left(\mathbb{R}_{+}\right):=\left\{f \in B_{\rho_{\gamma}}\left(\mathbb{R}_{+}\right): f \text { continuous on } \mathbb{R}_{+}\right\} \text {. }
$$

The norm in $B_{\rho_{\gamma}}$ is defined as

$$
\|f\|_{\rho_{\gamma}}=\sup _{x \in \mathbb{R}_{+}} \frac{|f(x)|}{\rho_{\gamma}(x)}
$$

We can give some estimations of the errors $\left|L_{n}^{*(\alpha, \beta)}(f ; q, x)-f(x)\right|, n \in \mathbb{N}$, for unbounded functions by using a weighted modulus of smoothness associated to the space $B_{\rho_{\gamma}}\left(\mathbb{R}_{+}\right)$.

We consider

$\Omega_{\rho_{\gamma}}(f ; \delta)=\sup _{x \geq 0,0<h \leq \delta} \frac{|f(x+h)-f(x)|}{1+(x+h)^{2+\gamma}}, \quad \delta>0, \quad \gamma \geq 0$.

It is evident that for each $f \in B_{\rho_{\gamma}}\left(\mathbb{R}_{+}\right), \Omega_{\rho_{\gamma}}(f ;$.$) is well$ defined and

$$
\Omega_{\rho_{\gamma}}(f ; \delta) \leq 2\|f\|_{\rho_{\gamma}}
$$

The weighted modulus of smoothness $\Omega_{\rho_{\gamma}}(f ;$.$) possesses$ the following properties.

$$
\begin{gathered}
\Omega_{\rho_{\gamma}}(f ; \lambda \delta) \leq(\lambda+1) \Omega_{\rho_{\gamma}}(f ; \delta), \delta>0, \lambda>0 \\
\Omega_{\rho_{\gamma}}(f ; n \delta) \leq n \Omega_{\rho_{\gamma}}(f ; \delta), n \in N \\
\lim _{\delta \rightarrow 0^{+}} \Omega_{\rho_{\gamma}}(f ; \delta)=0 .
\end{gathered}
$$

As it is known, weighted Korovkin type theorems have been proven by Gadjiev (see [10]).

Theorem 3.1. Let $q \in(0,1)$ and $\gamma \geq 0$. For all nondecreasing $f \in B_{\rho_{\gamma}}\left(\mathbb{R}_{+}\right)$we have

$\left|L_{n}^{*(\alpha, \beta)}(f ; q, x)-f(x)\right| \leq \sqrt{L_{n}^{*(\alpha, \beta)}\left(\mu_{x, \gamma}^{2} ; q, x\right)}\left(1+\frac{1}{\delta} \sqrt{L_{n}^{*(\alpha, \beta)}\left(\psi_{x}^{2} ; q, x\right)}\right) \Omega_{\rho_{\gamma}}(f ; \delta)$,

$x \geq 0, \quad \delta>0, \quad n \in \mathbb{N}, \quad$ where $\mu_{x, \gamma}(t):=1+(x+|t-x|)^{2+\gamma}, \quad \psi_{x}(t):=|t-x|, t \geq 0$.
Proof. Let $n \in \mathbb{N}$ and $f \in B_{\rho_{\gamma}}\left(\mathbb{R}_{+}\right)$. From (13) and (14), we can write

$$
\begin{aligned}
|f(t)-f(x)| & \leq\left(1+(x+|t-x|)^{2+\gamma}\right)\left(1+\frac{1}{\delta}|t-x|\right) \Omega_{\rho_{\gamma}}(f ; \delta) \\
& =\mu_{x, \gamma}(t)\left(1+\frac{1}{\delta} \psi_{x}(t)\right) \Omega_{\rho_{\gamma}}(f ; \delta) .
\end{aligned}
$$

Taking into account the definition of $q$ - integration, we get

$$
\int_{q\left(\frac{[k]_{q}+q^{k-1} \alpha}{[n]_{q}+\beta}\right)}^{\frac{[k+1]_{q}+q^{k} \alpha}{[n]_{q}+\beta}} f\left(q^{-k+1} t\right) d_{q} t=q^{k-1} \int_{\frac{[k]_{q}+q^{k-1} \alpha}{q^{k-2}\left([n]_{q}+\beta\right)}}^{\frac{[k+1]_{q}+q^{k} \alpha}{q^{k-1}\left([n]_{q}+\beta\right)}} f(t) d_{q} t .
$$

Consequently, the operators $L_{n}^{*(\alpha, \beta)}$ can be expressed as follows

$$
\begin{aligned}
L_{n}^{*(\alpha, \beta)}(f ; q, x)=( & {\left.[n]_{q}+\beta\right) \sum_{k=0}^{\infty} q^{\frac{k(k-1)}{2}} \frac{D_{q}^{k}\left(\varphi_{n}(x)\right)}{[k]_{q} !}(-x)^{k} q^{k-1} } \\
& \int^{\frac{[k+1]_{q}+q^{k} \alpha}{q^{k-1}\left([n]_{q}+\beta\right)}} f(t) d_{q} t .
\end{aligned}
$$

By using the Cauchy-Schwartz inequality and (15), we obtain

$$
\begin{aligned}
& \left|L_{n}^{*(\alpha, \beta)}(f ; q, x)-f(x)\right| \\
\leq & \left([n]_{q}+\beta\right) \sum_{k=0}^{\infty} q^{\frac{k(k-1)}{2}} \frac{D_{q}^{k}\left(\varphi_{n}(x)\right)}{[k]_{q} !}(-x)^{k} q^{k-1} \\
& \quad \int^{\frac{[k+1]_{q}+q^{k} \alpha}{q^{k-1}\left([n]_{q}+\beta\right)}}|f(t)-f(x)| d_{q} t \\
& \quad \int_{q^{k-2}\left([n]_{q}+\beta\right)}^{\frac{[k]^{k}+1}{k-\beta}} \\
\leq & \left(L_{n}^{*(\alpha, \beta)}\left(\mu_{x, \gamma} ; q, x\right)+\frac{1}{\delta} L_{n}^{*(\alpha, \beta)}\left(\mu_{x, \gamma} \psi_{x} ; q, x\right)\right) \Omega_{\rho_{\gamma}}(f ; \delta) \\
\leq & \sqrt{L_{n}^{*(\alpha, \beta)}\left(\mu_{x, \gamma}^{2} ; q, x\right)}\left(1+\frac{1}{\delta} \sqrt{L_{n}^{*(\alpha, \beta)}\left(\psi_{x}^{2} ; q, x\right)}\right) \Omega_{\rho_{\gamma}}(f ; \delta) .
\end{aligned}
$$

Lemma 3.2. For $m \in \mathbb{N}$ and $q \in(0,1)$ we have

$$
L_{n}^{*(\alpha, \beta)}\left(e_{m} ; q, x\right) \leq A_{m, q}\left(1+x^{m}\right), x \in \mathbb{R}_{+}, n \in \mathbb{N}
$$

where $A_{m, q}$ is a positive constant depending only on $m, \alpha$ and $q$.

Proof. For $k \in \mathbb{N}$ and $0<q<1$ the following inequality holds true

$$
1 \leq[k+1]_{q} \leq 2[k]_{q} .
$$


Thus, for $m \in \mathbb{N}$, from (1) and (16) we get

$$
\begin{aligned}
L_{n}^{\alpha, \beta}\left(e_{m} ; q, x\right)= & \sum_{k=0}^{\infty} q^{\frac{k(k-1)}{2}} \frac{D_{q}^{k}\left(\varphi_{n}(x)\right)}{[k]_{q} !}(-x)^{k} \frac{1}{q^{k m-m}}\left(\frac{[k]_{q}+q^{k-1} \alpha}{[n]_{q}+\beta}\right)^{m} \\
= & \frac{x[n]_{q}}{[n]_{q}+\beta} \sum_{k=0}^{\infty} q^{\frac{k(k-1)}{2}} \frac{D_{q}^{k}\left(\varphi_{m(n)}(x)\right)}{[k]_{q} !}(-x)^{k} \frac{1}{q^{k(m-1)}}\left(\frac{[k]_{q}+q^{k} \alpha}{[n]_{q}+\beta}\right)^{m-1} \\
& +\frac{\alpha}{[n]_{q}+\beta} \sum_{k=0}^{\infty} q^{\frac{k(k-1)}{2}} \frac{D_{q}^{k}\left(\varphi_{n}(x)\right)}{[k]_{q} !}(-x)^{k}\left(\frac{[k]_{q}+q^{k-1} \alpha}{q^{k-1}\left([n]_{q}+\beta\right)}\right)^{m-1} \\
\leq & \frac{x[n]_{q}}{[n]_{q}+\beta} \varphi_{m(n)}(x)\left(\frac{1+\alpha}{[n]_{q}+\beta}\right)^{m-1} \\
& +\frac{x[n]_{q}}{[n]_{q}+\beta} \sum_{k=0}^{\infty} q^{\frac{k(k-1)}{2}} \frac{D_{q}^{k}\left(\varphi_{m(n)}(x)\right)}{[k]_{q} !}(-x)^{k}\left(\frac{2[k]_{q}+q^{k} \alpha}{q^{k}\left([n]_{q}+\beta\right)}\right)^{m-1} \\
& +\frac{\alpha}{[n]_{q}+\beta} L_{n}^{\alpha, \beta}\left(e_{m-1} ; q, x\right) \\
= & \frac{x[n]_{q}}{[n]_{q}+\beta} \varphi_{m(n)}(x)\left(\frac{1+\alpha}{[n]_{q}+\beta}\right)^{m-1} \\
& +\frac{x[n]_{q}}{[n]_{q}+\beta}\left(\frac{2}{q}\right)^{m-1} L_{n+1}^{\alpha, \beta}\left(e_{m-1} ; q, x\right)+\frac{\alpha}{[n]_{q}+\beta} L_{n}^{\alpha, \beta}\left(e_{m-1} ; q, x\right)
\end{aligned}
$$

and we have

$$
\begin{aligned}
L_{n}^{\alpha, \beta}\left(e_{m} ; q, x\right) & \leq x+\left(\frac{2}{q}\right)^{m-1} \frac{1}{[n]_{q}+\beta}\left(x[n]_{q}+\alpha q^{m-1}\right) L_{n+1}^{\alpha, \beta}\left(e_{m-1} ; q, x\right) \\
& \leq 2 m\left(\frac{2}{q}\right)^{m-1} \frac{1}{[n]_{q}+\beta}\left([n]_{q}\left(1+x^{m}\right)+\alpha^{m} q^{\frac{m(m-1)}{2}}\right) .
\end{aligned}
$$

based on the above inequality and by using the mathematical induction over $m \in \mathbb{N}$, we obtain

$$
L_{n}^{\alpha, \beta}\left(e_{m} ; q, x\right) \leq B_{m, q}\left(1+x^{m}\right)
$$

$x \in \mathbb{R}_{+}, n \in \mathbb{N}$, where

$$
B_{m, q}:=2 m\left(\frac{2}{q}\right)^{\frac{m(m-1)}{2}}\left(1+\alpha^{m} q^{\frac{m(m-1)}{2}}\right)
$$

On the other hand,

$$
\begin{gathered}
L_{n}^{*(\alpha, \beta)}\left(e_{m} ; q, x\right)=\left([n]_{q}+\beta\right) \sum_{k=0}^{\infty} q^{\frac{k(k-1)}{2}} \frac{D_{q}^{k}\left(\varphi_{n}(x)\right)}{[k]_{q} !}(-x)^{k} \\
\frac{[k+1]_{q}+q^{k} \alpha}{[n]_{q}+\beta} \\
\int^{k\left(\frac{[k]_{q}+q^{k-1} \alpha}{[n]_{q}+\beta}\right)} e_{m}\left(q^{-k+1} t\right) d_{q} t \\
=\frac{[n]_{q}+\beta}{\left([n]_{q}+\beta\right)^{m+1}} \sum_{k=0}^{\infty} q^{\frac{k(k-1)}{2}} \frac{D_{q}^{k}\left(\varphi_{n}(x)\right)}{[k]_{q} !}(-x)^{k} \frac{q^{-k m+m}}{[m+1]_{q}} \\
\times\left\{\left([k+1]_{q}+q^{k} \alpha\right)^{m+1}-q^{m+1}\left([k]_{q}+q^{k-1} \alpha\right)^{m+1}\right\} .
\end{gathered}
$$

We consider

$$
\left([k+1]_{q}+q^{k} \alpha\right)^{m+1}-q^{m+1}\left([k]_{q}+q^{k-1} \alpha\right)^{m+1} .
$$

For $k \in \mathbb{N}$, one obtain

$$
\begin{aligned}
& \left([k+1]_{q}+q^{k} \alpha\right)^{m+1}-q^{m+1}\left([k]_{q}+q^{k-1} \alpha\right)^{m+1} \\
= & \left(\left([k+1]_{q}+q^{k} \alpha\right)^{m}+q\left([k+1]_{q}+q^{k} \alpha\right)^{m-1}\right. \\
& \left.\left([k]_{q}+q^{k-1} \alpha\right)+\ldots+q^{m}\left([k]_{q}+q^{k-1} \alpha\right)^{m}\right) \\
\leq & (m+1)\left([k+1]_{q}+q^{k} \alpha\right)^{m} \\
\leq & (m+1) 2^{m}\left([k]_{q}+q^{k} \alpha\right)^{m},
\end{aligned}
$$

hence, we can write

$$
\begin{aligned}
L_{n}^{*(\alpha, \beta)}\left(e_{m} ; q, x\right) \leq & \frac{\varphi_{n}(x)(m+1)(1+\alpha)^{m} q^{m}}{\left([n]_{q}+\beta\right)^{m}[m+1]_{q}} \\
& +\frac{2^{m}(m+1)}{[m+1]_{q}} L_{n}^{\alpha, \beta}\left(e_{m} ; q, x\right) \\
\leq & A_{m, q}\left(1+x^{m}\right),
\end{aligned}
$$

where $A_{m, q}:=\frac{(m+1)(1+\alpha)^{m} q^{m}}{[m+1]_{q}}+\frac{2^{m}(m+1)}{[m+1]_{q}} B_{m, q}$ and $B_{m, q}$ is given by (17).

Remark 3.3. Since any linear positive operator is monotone, from Lemma 3.2 we can easily see that $L_{n}^{*(\alpha, \beta)}(f ; q,.) \in B_{\rho_{\gamma}}\left(\mathbb{R}_{+}\right)$for each $f \in B_{\rho_{\gamma}}\left(\mathbb{R}_{+}\right), \gamma \in \mathbb{N}_{0}$.

Theorem 3.4. Let $f \in B_{\rho_{\gamma}}\left(\mathbb{R}_{+}\right)$be a non-decreasing function, then

$$
\left\|L_{n}^{*(\alpha, \beta)}\left(f ; q_{n}, .\right)-f\right\|_{\rho_{\gamma+1}} \leq K_{\gamma, q_{0}} \Omega_{\rho_{\gamma}}\left(f ; \delta_{n}\right),
$$

where $\delta_{n}:=\sqrt{\frac{[n]_{q_{n}}+1}{q_{n}\left([n]_{q_{n}}+\beta\right)}}$ and $K_{\gamma, q_{0}}$ is a positive constant independent on $f$ and $n$.

Proof. The identities (3)-(5) imply

$$
\begin{aligned}
L_{n}^{*(\alpha, \beta)}\left(\psi_{x}^{2} ; q_{n}, x\right)= & L_{n}^{*(\alpha, \beta)}\left((t-x)^{2} ; q_{n}, x\right) \\
= & \frac{[n]_{q_{n}}[m(n)]_{q_{n}}}{q_{n}\left([n]_{q_{n}}+\beta\right)^{2}} x^{2} \\
& +\frac{[n]_{q_{n}}\left([3]_{q_{n}}+q_{n}\left((1+3 \alpha)[2]_{q_{n}}+1\right)\right)}{[3]_{q_{n}}\left([n]_{q_{n}}+\beta\right)^{2}} x \\
& +\frac{q_{n}^{2}\left(1+3 \alpha+3 \alpha^{2}\right)}{[3]_{q_{n}}\left([n]_{q_{n}}+\beta\right)^{2}}-2 x\left\{\frac{[n]_{q_{n}}}{[n]_{q_{n}}+\beta} x+\frac{q_{n}(1+2 \alpha)}{[2]_{q_{n}}\left([n]_{q_{n}}+\beta\right)}\right\}+x^{2} \\
\leq & \frac{\left([n]_{q_{n}}+1+\beta\right) x^{2}}{q_{n}\left([n]_{q_{n}}+\beta\right)}+\frac{2(3 \alpha+3)}{q_{n}\left([n]_{q_{n}}+\beta\right)} x+\frac{1+3 \alpha+3 \alpha^{2}}{q_{n}\left([n]_{q_{n}}+\beta\right)} \\
\leq & \frac{9(1+\beta)^{2} \rho_{0}(x)}{q_{n}\left([n]_{q_{n}}+\beta\right)}\left\{[n]_{q_{n}}+1\right\}
\end{aligned}
$$

Let $\gamma \in \mathbb{N}_{0}$ and $f \in B_{\rho_{\gamma}}\left(\mathbb{R}_{+}\right)$be a fixed function. From 
Theorem 3.1 and above inequality, we can write

$$
\begin{aligned}
& \frac{\left|L_{n}^{*(\alpha, \beta)}\left(f ; q_{n}, x\right)-f(x)\right|}{\rho_{\gamma+1}(x)} \\
\leq & \sqrt{\frac{L_{n}^{*(\alpha, \beta)}\left(\mu_{x, \gamma}^{2} ; q_{n}, x\right)}{\rho_{\gamma+1}^{2}(x)}}\left(1+\frac{1}{\delta_{n}} \sqrt{L_{n}^{*(\alpha, \beta)}\left(\psi_{x}^{2} ; q_{n}, x\right)}\right) \Omega_{\rho_{\gamma}}\left(f ; \delta_{n}\right) \\
\leq & \sqrt{\frac{L_{n}^{*(\alpha, \beta)}\left(\mu_{x, \gamma}^{2} ; q_{n}, x\right) \rho_{0}(x)}{\rho_{\gamma+1}^{2}(x)}}\left(1+\frac{1}{\delta_{n}} \sqrt{\frac{9(1+\beta)^{2} \rho_{0}(x)}{q_{n}\left([n]_{q_{n}}+\beta\right)}\left\{[n]_{q_{n}}+1\right\}}\right) \Omega_{\rho \gamma}\left(f ; \delta_{n}\right) \\
\leq & 12(1+\beta) \sqrt{\frac{L_{n}^{*(\alpha, \beta)}\left(\mu_{x, \gamma}^{2} ; q_{n}, x\right)}{\rho_{2(\gamma+1)}(x)}}\left(1+\frac{1}{\delta_{n}} \sqrt{\frac{[n]_{q_{n}}+1}{q_{n}\left([n]_{q_{n}}+\beta\right)}}\right) \Omega_{\rho_{\gamma}\left(f ; \delta_{n}\right) .}
\end{aligned}
$$

Since

$$
\begin{aligned}
\mu_{x, \gamma}^{2}(t) & =\left(1+(x+|t-x|)^{2+\gamma}\right)^{2} \leq 2\left(1+(2 x+t)^{4+2 \gamma}\right) \\
& \leq 2\left(1+2^{4+2 \gamma}\left((2 x)^{4+2 \gamma}+t^{4+2 \gamma}\right)\right)
\end{aligned}
$$

from Lemma 3.2, we get

$$
L_{n}^{*(\alpha, \beta)}\left(\mu_{x, \gamma}^{2} ; q_{n}, x\right) \leq \lambda_{\gamma, q_{n}}^{2} \rho_{2(\gamma+1)}(x),
$$

where $\quad \lambda_{\gamma, q_{n}}^{2}=2^{5+2 \gamma}\left(2^{4+2 \gamma}+A_{4+2 \gamma, q_{n}}\right)$. Choosing $\delta_{n}:=\sqrt{\frac{[n]_{q_{n}}+1}{q_{n}\left([n]_{q_{n}}+\beta\right)}}$ and $K_{\gamma, q_{0}}:=24(1+\beta) \lambda_{\gamma, q_{0}}$, where $q_{0}:=\min _{n \in \mathbb{N}} q_{n}$, the proof is finished.

Remark 3.5. If $\lim _{n \rightarrow \infty} q_{n}=1$, then $\lim _{n \rightarrow \infty} \delta_{n}=0$, which yields that $\lim _{n \rightarrow \infty} \Omega_{\rho_{\gamma}}\left(f ; \delta_{n}\right)=0$. Therefore Theorem 3.4 gives the rate of convergence of $L_{n}^{*(\alpha, \beta)}$ to $f$.

\section{References}

[1] U. Abel, V. Gupta, An estimate of the rate of convergence of a Bezier variant of the Baskakov-Kantorovich operators for bounded variation functions, Demonstratio math., 36, 123136 (2003).

[2] A. Aral, V. Gupta, The $q$-derivative and application to $q$-Szász-Mirakyan operators, Calcolo, 43, 151-170 (2006).

[3] A. Aral, V. Gupta, On the Durrmeyer type modification of the $q$-Baskakov type operators, Nonlinear Analysis, 72, 1171-1180 (2010).

[4] Ç. Atakut, On Kantorovich-Baskakov-Stancu type operators, Bull. Cal. Math. Soc., 91, 149-156 (1999).

[5] D. Barbosu, Kantorovich-Stancu type operators, J. Inequal. Pure Appl. Math., 5, Article 53, 6 pp (2004).

[6] V. A. Baskakov, An example of a sequence of linear positive operators in the space of continuous, DAN, 113, 249-251 (1957).

[7] İ. Büyükyazıcı, Ç. Atakut, On Stancu type generalization of $q$-Baskakov operators, Mathematical and Computer Modelling, 52, $752-759$ (2010).

[8] R. A. DeVore, G. G. Lorentz, Constructive Approximation, Springer, Berlin, (1993).
[9] A. D. Gadjiev, Ç. Atakut, On approximation of unbounded functions by the generalized Baskakov operators, Trans. Acad. Sci. Azerb. Ser. Phys.-Tech. Math. Sci., Math. Mech., 23, 33-42 (2003).

[10] A. D. Gadjiev, Theorems of the type of P. P. Korovkin's theorems, Math. Zametki, 20, 781-786 (1976).

[11] N. K. Govil, V. Gupta, Convergence of $q$-Meyer-KönigZeller-Durrmeyer operators, Adv. Stud. Contemp. Math. (Kyungshang), 19, 97-108 (2009).

[12] V. Gupta, Z. Finta, On certain $q$-Durrmeyer type operators, Appl. Math. Comput., 209, 415-420 (2009).

[13] V. Gupta, C. Radu, Statistical approximation properties of $q$-Baskakov-Kantorovich operators, Cent. Eur. J. Math., 7, 809-818 (2009).

[14] S. C. Jing, H. Y. Fan, $q-$ Taylor's formula with its $q-$ remainder, Comm. Theoret. Phys., 23, 117-120 (1995).

[15] V. G. Kac, P. Cheung, Quantum Calculus, Universitext, Springer-Verlag, New York, (2002).

[16] N. I. Mahmudov, Statistical approximation of Baskakov and Baskakov-Kantorovich operators based on the $q$ - integers, Cent. Eur. J. Math., 8, 816-826 (2010).

[17] D. D. Stancu, Approximation of function by a new class of polynomial operators, Rev. Roum. Math. Pures et Appl., 13, 1173-1194 (1968).

[18] D. D. Stancu, Asupra unei generalizari a polinoamelor lui Bernstein (Romanian), Studia Universitatis Babeş-Bolyai, 14, 31-45 (1969).

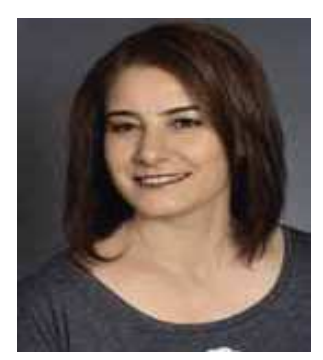

\section{Çiğdem Atakut} is an Lecturer in the Deparment of Mathematics, Faculty of Science, Ankara University, Ankara, Turkey. She received her Ph.D. from the Ankara University. Her research interest is on Approximation Theory

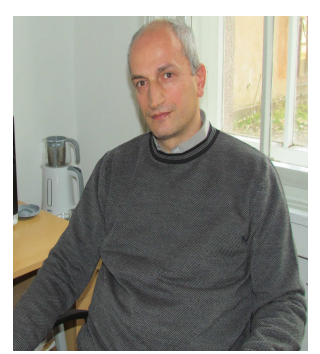

İbrahim Büyükyazıcı is an Associate Professor in the Department of Mathematics, Faculty of Science, Ankara University, Ankara, Turkey. He received his Ph.D. degree in Mathematics at Gazi University, Ankara in 2003. His research interest includes Approximation Theory. 